\title{
Optimal Channel Allocation with Hot-Spot Technique in Wireless Network
}

\author{
Smriti Dubey \\ Department of Computer Science and Engineering \\ Sati College, Vidisha (M.P)
}

\begin{abstract}
In wireless mobile communication systems, proper channel allocation scheme is required.

Due to the limitation of available frequency spectrum, channels must be reused as much as possible to increase system capacity. Complication arises in channel allocation, when more than one cell become "hot-spot" in the network of one BSC. The cells become "hot-spot" when bandwidth resources currently available in those cells are not sufficient to sustain any more call. In hybrid channel allocation, cells send a multilevel hot-spot notification to the dynamic pool of BSC on each channel request that cannot be satisfied locally at the base station. Proportional to the current hot-spot level of the cell, this hot-spot notification will request more than one channel to assign to the requesting cell. Severe complication arises, when not even one channel available in the dynamic pool of BSC in order to assign to requesting cells. This is the situation, when call starts dropping or blocking. This paper presents a new scheme in which BSC will fetch those ideal channels, which are borrowed to requesting cell from the dynamic pool in the time of hot spot but become ideal in the temporary pool of BTS for some period of time. BTS make use of these channels in order to sustain new incoming call The proposed algorithm in this paper will reduce the call dropping probability comparatively more than the simple hybrid channel allocation algorithm.
\end{abstract}

\section{Keywords:}

hybrid channel allocation, hot-spot, blocking probability, handover

\section{INTRODUCTION}

Due to increased urge to use the wireless communication in a satisfied way, a promised quality of service is required to manage incoming new calls and handoff more efficiently [2]. In radio resource management for wireless network, proper channel allocation scheme are required to allocate bandwidth for communication channels to base stations, access points and terminal equipment. Call refusal rate or Call drop rate is the main evaluation parameter, when talking about channel allocation scheme i.e. the ratio between numbers of drop calls to the total number of calls. For the given technique Call drop rate can be measured in erlang, where 1 erlang= 1 ongoing call per second. In [5\&6] different channel allocation algorithms are used to allocate channels. As in [1-3], Following are the three major categories for assigning channels to cells.

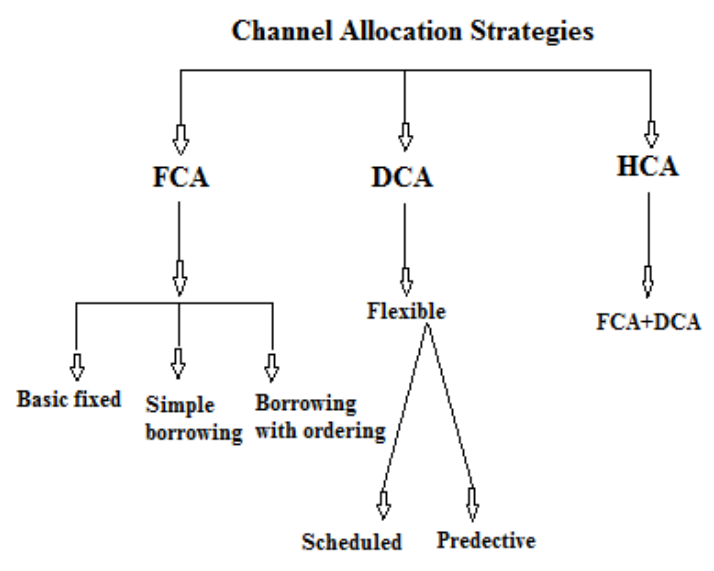

Fig .1 Channel Allocation Strategies

FIXED CHANNEL ALLOCATION (FCA):

In FCA, according to predetermined traffic demand and cochannel interference constraints, a fixed number of channels are assigned to each cell. [1] FCA is very simple but this scheme is difficult to survive in changing traffic conditions and user distributions. Over the whole service area if traffic varies from cell to cell, FCA could not attain a high efficiency of total channel usage. DCA strategy has been proposed, in order to overcome the problems of FCA.

\section{DYNAMIC CHANNEL ALLOCATION (DCA):}

In DCA, channels are not allocated to cells permanently. For every call request base station request channel from BSC. According to the duration of the calls, channels are assigned temporarily for use in cells. The channel is returned back and kept in the central pool, after the call is over. In order to avoid co-channel interference, any channel that is in use in one cell can only be reassigned to another cell simultaneously if the distance between those two cells is larger than the minimum reuse distance. DCA needs many transceivers for each base station. Behave well in low load but behave worse than FCA in heavy load. HCA strategy has been proposed, in order to overcome the problems of DCA. DCA schemes are suggested for TDMA/FDMA based cellular systems such as GSM, but are currently not used in any products.

\section{HYBRID CHANNEL ALLOCATION (HCA):}

The third category of channel allocation is hybrid channel allocation; it is hybrid of fixed and dynamic channel allocation algorithm. Channels in HCA are divided into two disjoint sets-

First is fixed set: set of channel which is assigned to each cell on FCA basis.

Second is dynamic set: set which are kept in a central pool of BSC for dynamic assignment. 
Fixed set channels are preferred for use in their respective cells, which are assigned to cells as in the FCA schemes. When a mobile user demands for a channel for its call, channel is assigned from fixed set, but when all the channels in its fixed set are busy, only then a request is made from dynamic set of central pool. The ratio of the fixed channels and dynamic channels plays a vital role in channel allocation scheme, if the ratio is $50 \%$ or more, than FCA performs better than HCA as in [1].

In this paper, we will investigate and propose a novel hybrid channel allocation scheme for wireless cellular networks, in terms of hot spot. The proposed scheme is simple to implement and give better result than simple hybrid channel allocation scheme [6] by decreasing calls drop probability. We have organized our discussion as follows. In section 2 we discussed previously work done in the field hybrid channel allocation. In section 3 we described our proposed algorithm, which comprises of the concept under taken. In section 4 we described the simulation method and result and in section 5 we conclude with a summary of what we have achieved.

\section{RELATED WORK:}

HCA performs better than any other channel allocation schemes in terms of call dropping and blocking by using hotspot notification and central pool [3]. When new call arrives in hot-spot cell than automatically a channel is assigned to that call from central pool till traffic in the cell goes to normal level. In heavy traffic load HCA is capable to minimize call blocking probability.

A level is maintained i.e. $\mathrm{L}=0$ (where $\mathrm{L}$ is hot-spot level) of the cell in the beginning to indicate that the channel request can be accommodated from the fixed set and there is no hotspot cell in the network. Now when a mobile user wants to connect a call, it request for a channel from its base station. If the base station has channels available in fixed set, it will assign channel to incoming channel request. But if no channel available in fixed set, then base station update its level by $1 \&$ sends a channel request to the central pool in order to borrow channels from BSC. Channel request include base station current level and maximum level. On receiving channel request from base station BSC will assign channels from the central pool to base station up to the requesting level at the same time base station add the same number of channel to its temporary pool. As the traffic increases, base station maintains its level on each channel request. When a call terminates base station check the type of channel the call belonged to. If the channel belonged to fixed set then it remain in base station but if channel belonged to dynamic pool, now base station is required to estimate or check its current level, if its level is below hot-spot level than channels are retained to BSC from its temporary pool otherwise remain in base station itself.

If the base station level is greater or equal to hot-spot level, meaning that the congestion in the cell is same or getting worse, now the channels remain in the cell itself in the temporary pool of base station in order to sustain more calls. Condition becomes critical when BSC has no channel available in its central pool and channel request arrives from any cell. In this case, call starts dropping or blocking.

\section{THE PROPOSED WORK:}

\subsection{System model:}

The system uses a hybrid channel allocation scheme where as in [3]; the total number of $\boldsymbol{C}$ channels is divided into two disjoint sets, $\boldsymbol{F}$ and $\boldsymbol{D}$. The set $\boldsymbol{F}$ contains the channels for fixed (or static) assignment, while the set $\boldsymbol{D}$ contains the channels for dynamic assignment, i.e., $\boldsymbol{C}=\boldsymbol{F} \mathrm{U} \boldsymbol{D}$. Moreover, each base station maintains a temporary pool (called $\boldsymbol{T}$ ) to retain a channel that was originally transferred from the dynamic assignment pool at BSC. The system uses a frequency reuse factor $N$. The fixed channels are assigned to a cell statically as in FCA; while dynamic channels are kept in a centralized pool at BSC. Let $\boldsymbol{r}$ be the ratio of the number of dynamic channels to the total number of channels available in the system, [13] i.e.

$$
\boldsymbol{r}=|\boldsymbol{D}| /|\boldsymbol{C}|
$$

The value of $r$ depends upon the traffic condition as well as on designer's view.

The "hot-spot" notification level is a multiple of 2 , such that $2^{i}$ Where $\mathrm{i}$ is an integer i.e. $0,1,2,3 \ldots \ldots$

$\mathrm{L}=\{1,2,4,8 \ldots . M\}$

Where $M=$ pre-defined maximum level supported by the system.

\& $\quad \mathbf{L}=$ represents the fact that up to $\mathbf{L}$ borrowed channels can be retained by the base station after a call on the borrowed channel from that cell terminates.

\subsection{Proposed algorithm:}

This algorithm consists of two phase i.e. channel acquisition and channel release phase. The following Steps are taken from Mobile Host, Base Station and MSC sides during both phases:

1. When any mobile host wants to connect a call, it searches for a channel related to its base station.

2. If base station has channel available in its fixed set, it will provide to the user and channel acquisition phase completed.

3. In case when no channel available in fixed set of that particular base station, it will create channel request from BSC.

It also includes the current value of $\boldsymbol{L}$ in the channel request; and the maximum value of $\boldsymbol{L}$ is a predefined number $\boldsymbol{M}$.

4. When the base station successfully acquires a channel from the dynamic pool at BSC, it also adds the channel to its temporary pool $(\boldsymbol{T})$.

5. When call terminates of that channel, then base station checks whether that particular channel belongs to fixed set or from dynamic pool. If from fixed set, then it will remain in base station but if it belongs to dynamic set then base station checks its current level L \& compare with its hot spot level (h).if $\mathrm{h}<\mathrm{L}$, then channels which are borrowed are return back to MSC. And if $h>=L$, then base station retain the channel according to its current level or according to its requirement.

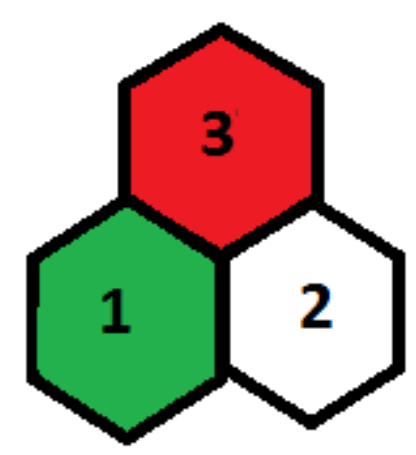

Fig 2. When two cells are hot-spot 
7. When traffic increases in the network, more cells become hotspot and borrow channels from MSC, now if any channel request arrives and MSC has no channel available in dynamic pool.

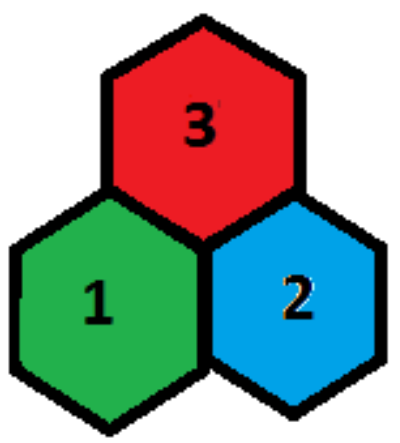

Fig 3. When all the cells are hot-spot in the network

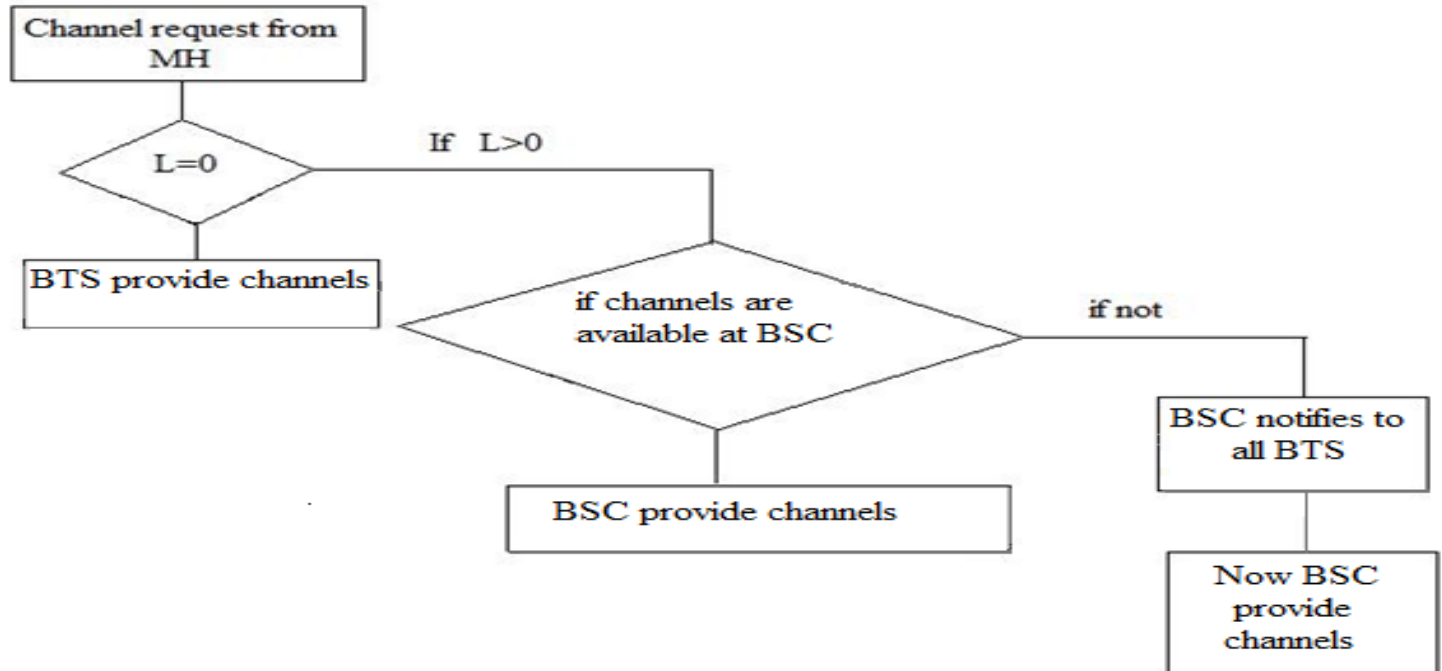

Fig 4. Flowchart for Channel Acquisition Phase

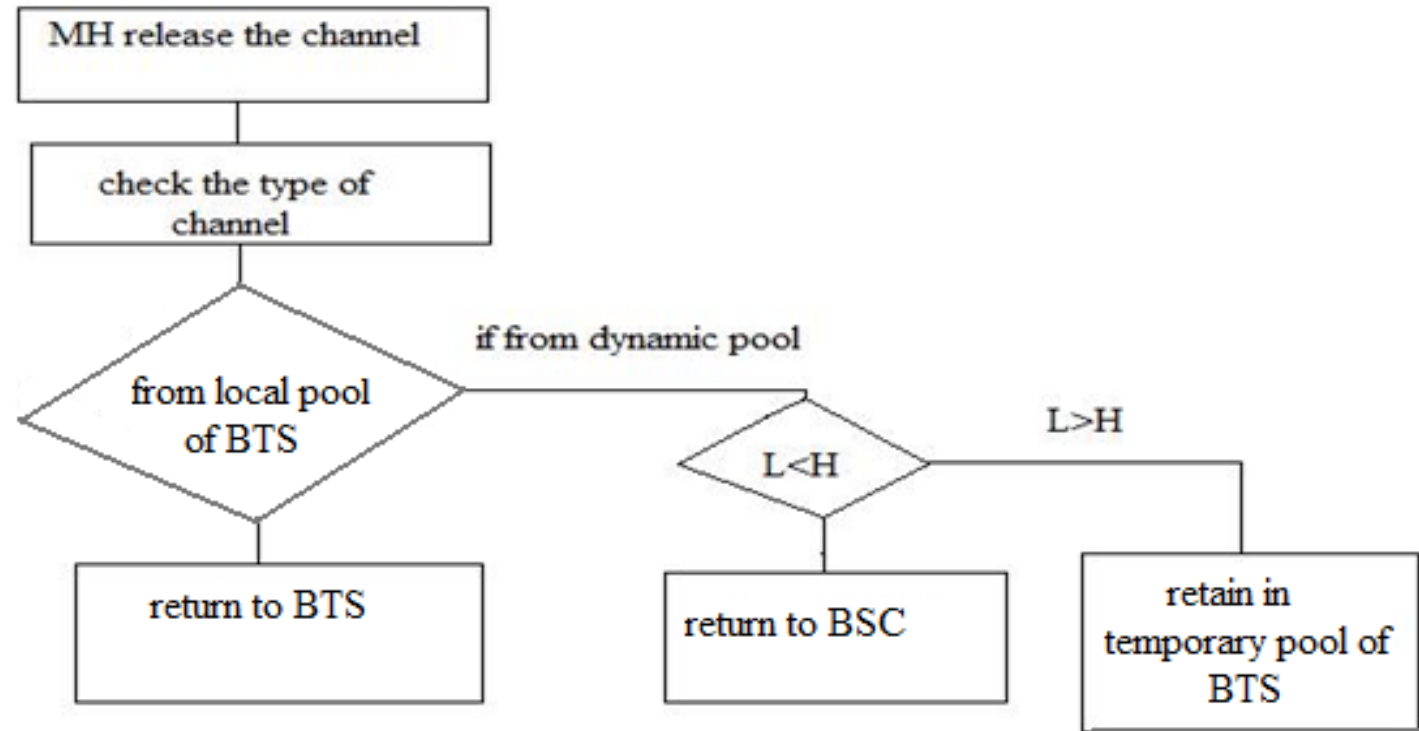

Fig 5. Flowchart for Channel Release Phase 


\section{SIMULATION:}

Many calculations are made to evaluate and compare the performance of the proposed algorithm of hybrid channel allocation with the existing one. In this paper, Parameters evaluated from Hybrid Channel Allocation using hot-spot notifications are as follows:

(1) Successful call percentage

(2) Call drop rate

(3) Call block rate

(4) Handover rate

The above parameters can be calculated as follows:

$$
\begin{aligned}
& \text { Successful call percentage }=\frac{\text { success calls } * 100}{\text { total calls }} \\
& \text { Call drop rate }=\frac{\text { no.of drop calls }}{\text { total calls }} \\
& \text { Call block rate }=\frac{\text { no.of block calls }}{\text { total calls }}
\end{aligned}
$$

The main objective of the proposed system is to obtain decrease no. of call drop rate, call block rate and handover. Percentage of call drop or block is expected to be low as compared to the classical hybrid channel allocation.

\subsection{Parameters:}

In this paper, simulated cellular network has 300 channels total available in the network. The ratio $r$ is varied from 0 to 0.8 .

Where $\quad r=$ number of dynamic channels/total number of channel available.

The system load (or traffic) intensity is defined with respect to arrival rates and call service rate in a cell that can switch back and forth from "normal" to "hot-spot" state. The value of $\boldsymbol{M}$ (the maximum "hot-spot" level supported by the system) is also varied from 4 to 8 .

\section{RESULT:}

We simulate the hybrid channel allocation in terms of blocking rate, dropping rate, handover calls versus traffic load by using OMNET 4.2.

Table 1, 2 \& 3 shows call block rate, call drop rate and handover calls rates

In fig 6, graph is plotted between system load and call block rate. According to this graph call block rate decreases when we increase the dynamic channel ratio to total channels. It is obvious that as the number of dynamic channel increases, call will be fulfilled but through our proposed method call block is reduced at greater percentage. Similarly in fig 7, call drop rate is reduced. Since BSC fetch channels from temporary pool in the time channel request, result in decrease of call drop rate. In the case of handover from fig 8 , we observed that when the system load is low, handover occurs more in all the three cases. But as the system load increases, then handover decreases in the case of 240 dynamic channels whereas in the FCA handover not decreases after sometime.

So in all the result we observed that as the ratio of dynamic channels increases, in all aspect performance becomes good. For the better communication system, it is required to vary the number of dynamic channels as per the traffic grows. For low traffic condition, even FCA performs better. Proper management system should to be maintaining in the time of high traffic and in the time of hot-spot to manage the entire mobile user.

\begin{tabular}{|l|ll|l|l|l|}
\hline \multirow{2}{*}{ System load } & \multicolumn{4}{|c|}{ Call blocked rate } \\
\cline { 2 - 5 } & & $\mathrm{fca}$ & $\mathrm{h}=0.1$ & $\mathrm{~h}=0.5$ & $\mathrm{~h}=0.8$ \\
\hline 0.25 & 0 & 0 & 0 & 0 \\
\hline 0.5 & 1.48 & 1.59 & 0.26 & 0.22 \\
\hline 0.75 & 5.20 & 4.99 & 4.39 & 3.21 \\
\hline 1 & 13.53 & 10.28 & 8.48 & 6.59 \\
\hline 1.25 & 18.41 & 16.09 & 15.66 & 10.88 \\
\hline
\end{tabular}

Table 1. Call block rate

\begin{tabular}{|c|c|c|c|c|}
\hline \multirow{2}{*}{$\begin{array}{c}\text { System } \\
\text { load }\end{array}$} & \multicolumn{4}{|c|}{ Call drop percentage } \\
\cline { 2 - 5 } & fca & $\mathrm{h}=0.1$ & $\mathrm{~h}=0.5$ & $\mathrm{~h}=0.8$ \\
\hline 0.25 & 0 & 0 & 0 & 0 \\
\hline 0.5 & 4.81 & 5.31 & 4.74 & 4.22 \\
\hline 0.75 & 14 & 13.75 & 12.61 & 11.83 \\
\hline 1 & 19.70 & 22.03 & 9.71 & 20.43 \\
\hline 1.25 & 22.30 & 25.30 & 24.88 & 8.98 \\
\hline
\end{tabular}

Table 2. Call drop rate 


\begin{tabular}{|c|c|c|c|c|}
\hline System load & \multicolumn{4}{|c|}{ Handover Calls } \\
\cline { 2 - 5 } & fca & $\mathrm{h}=0.1$ & $\mathrm{~h}=0.5$ & $\mathrm{~h}=0.8$ \\
\hline 0.25 & 1284.64 & 1287.31 & 1287.31 & 1287.31 \\
\hline 0.5 & 1145.52 & 1156.34 & 1212.10 & 1220 \\
\hline 0.75 & 1000 & 930.68 & 946.83 & 990.27 \\
\hline 1 & 615.23 & 645.36 & 1085.88 & 870.10 \\
\hline 1.25 & 502.61 & 467.34 & 577.76 & 998.20 \\
\hline
\end{tabular}

Table 3. Handover calls

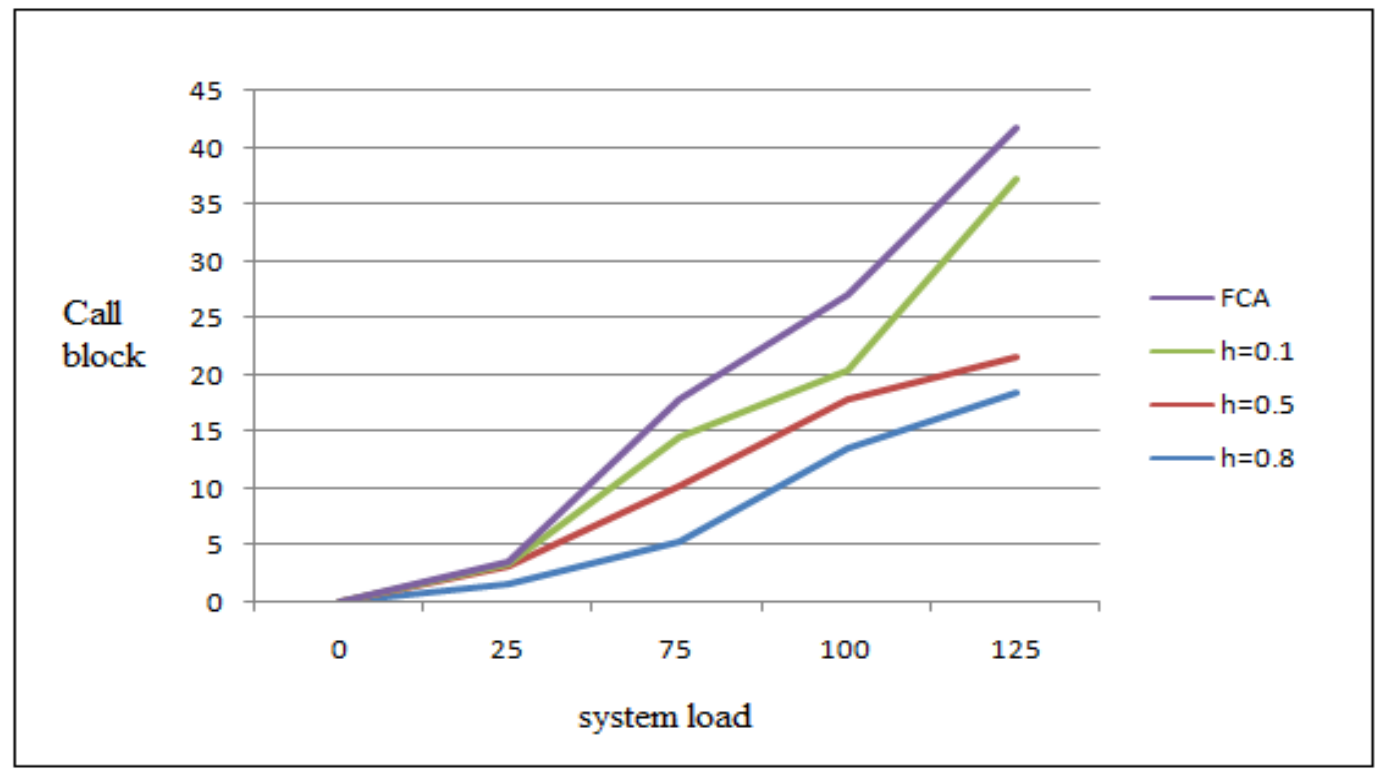

Fig 6. Graph showing call block versus system load

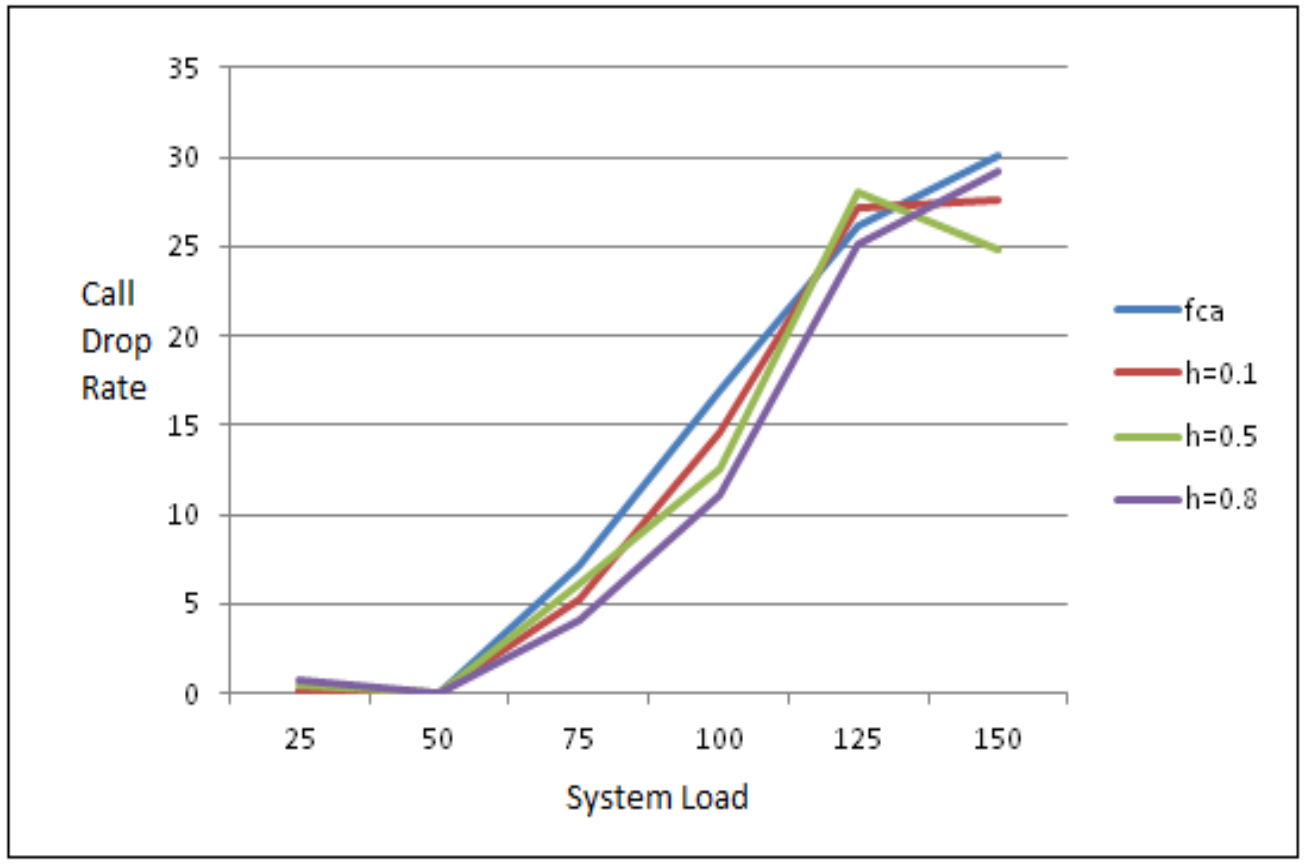

Fig 7. Graph showing call drop versus system load 


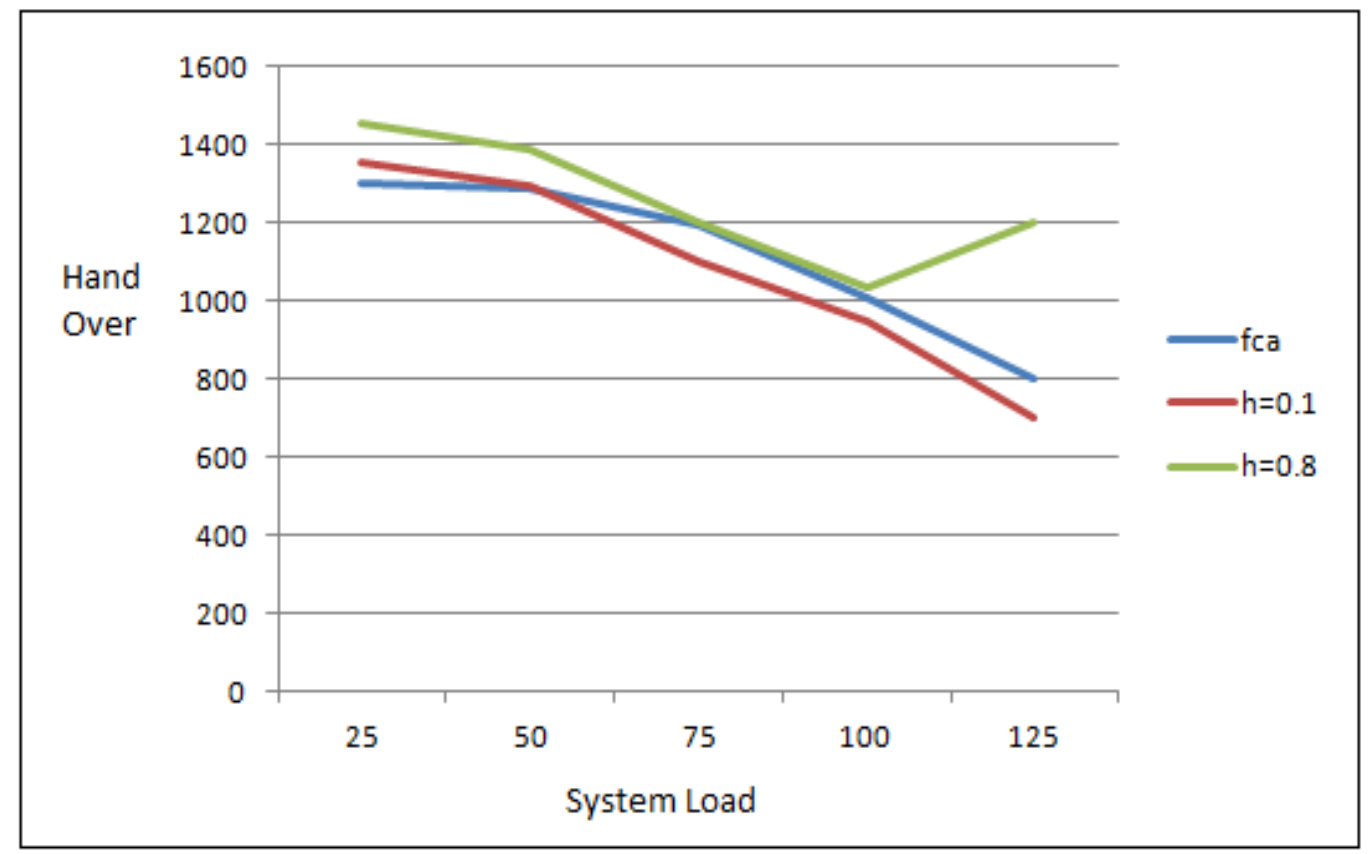

Fig 8. Graph showing handover calls versus system load

\section{CONCLUSION:}

The demand in the mobile communication has remarkably grown in the last two decade. An efficient channel allocation is essential to achieve the good performance in cellular system. While the limited number of channels available, requires efficient reuse of channels. To resolve this problem various channel allocation scheme developed by researchers. The present work is carried in the general context of channel allocation issue involved in cellular networks at heavy load situations. The simulation of this approach is expounded and evaluated over OMNeT++ in a scenario with fixed channel allocation and hybrid approach by varying the proportion of dynamic channels to total number of channels available and the effectiveness is evaluated in terms of Call blocked and Call dropped versus System load. The work presented here illustrates the efficiency and flexibility of hybrid channel allocation algorithm along with a slight modification in conventional algorithm by introducing the channel retrieval phase which retrieves back the channels with BS, borrowed from MSC and are unused and allocates the same to some BS requiring it and thus resolving the limitation of MSC of being exhausted of communication bandwidth to serve further calls in heavy load. The call effectiveness of the work has been illustrated in simulation where the approach performs better in almost all cases as compared to its fixed counterparts.

\section{REFERENCES}

[1] M.L.S.N.S. Lakshmi, M.S.L. Ratnavathi and S. Gopi Krishna,"An insight to call blocking probabilities of channel assignment schemes" International Journal of Advances in Engineering \& Technology, May 2012.

[2] S. Malathy and G. Sudha Sadasivam, "Design of Optimal Channel Allocation for Mobile Users in Cellular Networks", European Journal of Scientific Research ISSN 1450-216X Vol.69 No.4 (2012), pp. 599-606 () EuroJournals Publishing, Inc. 2012
[3] Rana Ejaz Ahmed, "A channel allocation algorithm using hot-spot notification for wireless cellular networks" IEEE CCECE/CCGEI, Ottawa, May 2006

[3] Rana Ejaz Ahmed, "A Channel Allocation Algorithm for Hot-Spot Cells in Wireless Networks", Journal of advances in information technology, vol 1,no. 3,august 2010.

[4] Geetali Vidyarthi, Alioune Ngom, and Ivan Stojmenovic, "A Hybrid Channel Assignment Approach Using an Efficient Evolutionary Strategy in Wireless Mobile Networks", IEEE TRANSACTIONS ON VEHICULAR TECHNOLOGY, VOL. 54, NO. 5, SEPTEMBER 2005

[5] Y.S.V.Raman1, G.Sricharan2, G.Naveen Kumar2, B.Siddardha2, " The Performance Evaluation of Hybrid Channel Allocation Strategy in Cellular Networks", International Journal of Engineering Research and Applications (IJERA) ISSN: 2248-9622 may-jun 2012

[6] Jiming Chen, Senior Member, IEEE, Qing Yu, Peng Cheng, Youxian Sun, Yanfei Fan, and Xuemin Shen, Fellow, IEEE, "Game Theoretical Approach for Channel Allocation in Wireless Sensor and Actuator Networks", IEEE transactions on automatic control, vol. 56, no. 10, october 2011

[7] Mohamed Kadhem Karray and Yasir Khan, "Evaluation and comparison of resource allocation strategies for new streaming services in wireless cellular networks", 19781-4673-1008-6/12/\$31.00 c 2012 IEEE

[8] G"unter Reise and Gerald Matz, "Optimal transmit power allocation in wireless sensor networks performing field reconstruction" , 978-1-4673-0046-9/12/\$26.00 (C)2012 IEEE

[9] Fan $\mathrm{Wu}$ and Nitin Vaidya, "Workload-Aware Opportunistic Routing in Multi-Channel, Multi-Radio Wireless Mesh Networks", 2012 9th Annual IEEE 
Communications Society Conference on Sensor, Mesh and Ad Hoc Communications and Networks (SECON)

[10] Prof. Dr.-Ing. Jochen Schiller, http://www.jochenschiller.de/ MC SS05, "Mobile Communications Chapter 2: Wireless Transmission"

[11] Yu cheng, hongkun li, and peng-jun wan, "A Theoretical framework for optimal cooperative networking in multiradio multichannel wireless networks", IEEE Wireless Communications • April 2012

[12] Ashish Raniwala, Kartik Gopalan and Tzi-cker Chiueh, "Centralized Channel Assignment and Routing Algorithms for Multi-Channel Wi reless Mesh Networks", Mobile Computing and Communications Review, Volume 8, Number 2

[13] J Joshi, G Mundada, "A hybrid channel allocation algorithm to reduce call blocking probability using hotspot notification", Information and Automation for sustainability, 2010 - ieeexplore.ieee.org
[14] Hasan C, am, "Nonblocking OVSF Codes and Enhancing Network Capacity for 3G Wireless and Beyond Systems", Proc. of The 2002 International Conference on Wireless Networks, June 24-27, 2002, Las Vegas, USA

[15] Habiba Skalli, IMT Lucca Institute for Advanced Studies Samik Ghosh and Sajal K. Das, The University of Texas at Arlington, "Channel Assignment Strategies for Multi radio Wireless Mesh Networks: Issues and Solutions." IEEE Communications Magazine • November 2007

[16] Wei Wang and Xin Liu," List-coloring Based Channel Allocation for Open-Spectrum Wireless Networks", University of California, Davis Davis, CA 95616, U. S. A.

[17] Ray -Guang Cheng \& phone Lin, "OVSF Code Channel Assignment For IMT-2000” 0-7803-5718-3/00\$10.00 2000 IEEE. 